\title{
Tipos de circulação e predominância das artérias coronárias em corações de brasileiros
}

\author{
Renato FALCI JÚNIOR*, Richard Halti CABRAL*, Nadir Eunice Valverde B. de PRATES** \\ RBCCV 44205-210
}

FALCI JÚNIOR, R.; CABRAL, R. H.; PRATES, N. E. V. B. - Tipos de circulação e predominância das artérias coronárias em corações de brasileiros. Rev. Bras. Cir. Cardiovasc., 8(2):152-162, 1993.

RESUMO: O conhecimento anatômico do tipo de circulação, com a identificação da dominância das artérias coronárias na irrigação do coração, apresenta grande interesse clínico e cirúrgico, devido a que variações nessa irrigação ocasionam diferentes graus de gravidade em casos de obstrução. Foram estudados 50 corações retirados de indivíduos adultos, de ambos os sexos e diferentes raças, nos quais as artérias coronárias foram dissecadas, visando identificar o tipo de circulação. Realizamos, ainda, estudo morfométrico em corações cujo peso médio foi 291 gramas e altura ventricular média de $97 \mathrm{~mm}$. Em $72 \%$ dos corações estudados havia dominância da direita, $16 \%$ circulação balanceada e $12 \%$ dominância da esquerda. Identificamos o número de ramos que ultrapassam a crux cordis, sendo o mínimo de um ramo e o máximo de cinco ramos, com valor médio de 2,2 nos casos de dominância da direita e em apenas dois corações um ramo (em cada um) nos 8 de dominância da esquerda. Em $50 \%$ dos corações estudados o ramo interventricular anterior ultrapassa o ápice cardiaco, atingindo a sua face diafragmática.

DESCRITORES: artérias coronárias, morfologia; artérias coronárias, anatomia.

\section{INTRODUÇÃO}

O estudo morfológico das artérias coronárias sempre foi um assunto de grande interesse na anatomia e na cardiologia. Devido à grande variabilidade na distribuição das artérias no coração e possíveis correlações anatomoclínicas entre o tipo de irrigação e o diferente risco de infarto do miocárdio, as artérias coronárias vêm sendo melhor estudadas ultimamente, com a difusão da cinecoronariografia.

"É de grande interesse saber o porquê das variações e as leis que governam as variaçōes das artérias coronárias". Nesta frase célebre que BANCHI 1 cita em trabalho sobre a morfologia das artérias coronárias, abre para a pesquisa um novo capítulo da anatomia. $O$ autor enfatiza $\circ$ fato de que as variações da irrigação do coração dependem da relação recíproca entre o comprimento do ramo circunflexo da artéria coronária esquerda e o da artéria coronária direita.

Em 1929 CAMPBELL ${ }^{4}$, em estudo radiológico das artérias coronárias, descreve as variaçōes na sua distribuição ventricular. $\mathrm{Na}$ superfície anterior do coração há pequenas variações, sendo o ramo interventricular anterior um ramo constante da artéria coronária esquerda. No entanto, na superfície posterior, há variações no tamanho e na distribuição. Para o autor, a artéria coronária direita é rudimentar em $18 \%$ dos casos e envia apenas um

Trabalho realizado no Departamento de Anatomia do Instituto de Ciências Biomédicas e Faculdade de Medicina da Universidade de São Paulo. São Paulo, SP, Brasil.

Recebido para publicação em 30 de março de 1993.

- Acadêmico da Faculdade de Medicina da USP.

* Professora do Departamento de Anatomia do Instituto de Ciências Biomédicas.

Endereço para separatas: Renato Falci Júnior. Faculdade de Medicina - USP. Av. Dr. Arnaldo, 455. 01423-906 São Paulo, SP, Brasil. 
FALCIJÚNIOR, R.; CABRAL, R. H.; PRATES, N. E. V.B. - Tipos de circulação e predominância das artérias coronárias em corações de brasileiros. Rev. Bras. Cir. Cardiovasc., 8(2):152-162, 1993.

ramo para a face posterior do ventrículo direito em $54 \%$ dos casos. Tem uma distribuição equilibrada com a artéria coronária esquerda em $14 \%$ e atravessa a crux cordis em $14 \%$ dos casos.

Já WHITTEN ${ }^{15}$ dá ênfase especial ao aspecto clínico, estudando a relação da distribuição e estrutura das artérias coronárias com o infarto do miocárdio. Descreve que em 47 casos de infarto do miocárdio havia acometimento do ventrículo esquerdo em todos; em 36 deles o ventrículo esquerdo recebia suprimento da artéria coronária esquerda; em 22 casos havia participação da artéria coronária direita e em apenas 4 casos havia infarto do ventrículo direito. $\mathrm{O}$ autor relata, ainda, que o infarto do ventrículo esquerdo por lesão da artéria coronária esquerda é mais freqüente por doença do ramo interventricular anterior que do ramo circunflexo e o fato de que o infarto é mais freqüente no ventrículo esquerdo do que no direito parece depender das diferenças anatômicas de distribuição das artérias coronárias nos dois ventrículos.

Em 1931, EHRICH et alii ${ }^{5}$ estudam as artérias coronárias em 51 corações humanos de 0 a 97 anos, através de métodos radiológicos, anatômicos e histológicos. Descrevem o trajeto macroscópico das artérias coronárias e suas alterações em relação à idade. Enfatizam que, em alguns casos, a artéria coronária direita é pequena e termina na superfície posterior entre a margem direita e a crux cordis e, em outros, ela pode ser mais longa, extendendo-se até a margem esquerda. Descrevem distribuição semelhante para a artéria coronária esquerda. Os autores também demonstram a existência de diferenças notáveis entre as artérias coronárias direita e esquerda, sendo que os ramos da esquerda, embora presentes em menor número, possuem comprimento maior, tendendo a atingir o ápice do coração; concluem que o desenvolvimento das duas artérias coronárias é paraticamente igual.

SCHLESINGER ${ }^{11}$ introduz o conceito de dominância, baseado no suprimento arterial da crux cordis, que corresponde à região posterior do coração onde os sulcos atrioventricular, interatrial e interventricular se encontram. Com base nesse critério, o autor relata três tipos de circulação coronariana: balanceada, dominância de direita e dominância de esquerda. Considera circulação balanceada quando ambas as artérias atingem a crux cordis e não a ultrapassam, sendo o ramo interventricular posterior ramo da artéria coronária direita. Se esta artéria atravessa a crux cordis e dá um ou mais ramos para o ventrículo esquerdo, o autor refere a dominância de direita e, finalmente, quando o ramo circunflexo da artéria coronária esquerda origina o ramo interventricular posterior e, facultativamente, dá algum ramo para o ventrículo direito, considera dominância de esquerda.
O mesmo SCHLESINGER ${ }^{12}$ estuda a relação entre o padrão anatômico e as condições patológicas das artérias coronárias, em 269 corações humanos. Como as oclusões de ramos das artérias coronárias estavam ausentes até a idade de 37 anos, o autor decide estudar o padrão anatômico normal em 225 corações com idade superior a 35 anos. Encontra dominância de direita em $48 \%$, balanceada em $34 \%$ e dominância de esquerda em $18 \%$. Refere, ainda, que os infartos que ocorrem nos corações com circulação balanceada são mais benignos, raramente levando à morte, concluindo que o tipo de circulação balanceada é o melhor padrão para o ser humano. Por outro lado, os corações com dominância de esquerda constituem um grupo patologicamente importante, pois são os que mais sofrem com a arteriosclerose $e$, conseqüentemente, com o infarto do miocárdio.

Em 1940, BLUMGART et alii ${ }^{3}$ estudam a importância das variações na distribuição anatômica das artérias coronárias, observando 125 corações. Definem três tipos de circulação: balanceada, dominância de direita e dominância de esquerda, encontrado uma estatística de $40 \%, 40 \%$ e $20 \%$, respectivamente.

Em 1951, ZOLL 16 estuda as anatomias normal e patológica das artérias coronárias usando técnica radiológica, a qual serve de guia para dissecção e análise das anastomoses em corações normais e de cardiopatas. Em relação à irrigação, relata a dominância de esquerda como grupo muito pouco freqüente (1/6 dos casos), circulação balanceada, e constata cerca de $50 \%$ de dominância de direita. Para o autor, as variações anatômicas são de considerável importância clínica e patológica.

Estudando as variações da circulação coronária, FANFANI ${ }^{7}$ analisa 150 corações de indivíduos com idade variável entre 30 e 80 anos, através da técnica radiológica. Encontra $116(77,33 \%)$ casos de dominância de direita, $24(16 \%)$ casos de dominância de esquerda e $10(6,67 \%)$ casos de circulação balanceada.

Em 1958, SPADA et alii ${ }^{13}$, dedicando-se ao estudo da anatomia radiológica das artérias coronárias, identificam e definem dois tipos de circulação, baseados no comprimento do ramo circunflexo. No primeiro grupo, o ramo circunflexo é curto e o ramo interventricular posterior é ramo da artéria coronária direita; no segundo grupo, o ramo circunflexo é longo e origina o ramo interventricular posterior. Referem que a região do miocárdio mais propensa à insuficiência coronária e infarto é constituída pelo território dependente do ramo interventricular anterior, sendo a artéria coronária esquerda denominada artéria da morte súbita.

MAY ${ }^{9}$ publica trabalho sobre a anatomia cirúr- 
FALCIJÚNIOR, R.; CABRAL, R. H.; PRATES, N. E. V.B. - Tipos de circulação e predominância das artérias coronárias em coraçōes de brasileiros. Rev. Bras. Cir. Cardiovasc., 8(2):152-162, 1993.

gica das artérias coronárias. Estuda radiologicamente 500 corações não formolizados obtidos em necropsia. Encontra dominância de direita em 55\% dos casos, balanceada em $9 \%$ e dominância de esquerda em $36 \%$. Na maioria dos casos, a artéria coronária direita dá, em média, 1,8 ramos para o ventrículo esquerdo, apresentando um máximo de seis ramos. Encontra, em média, número semeIhante de ramos para os dois ventrículos, embora - ventrículo esquerdo necessite de cerca de duas vezes mais suprimento sangüíneo, independente da dominância, o que é esperado devido ao diferente papel fisiológico de cada ventrículo.

Em 1961, VASKO et alii ${ }^{14}$ estudam dominância, das artérias coronárias através de arteriografia e perfusão em 56 corações humanos com idade de 7 a 93 anos, sem cardiopatias agudas. No estudo anatômico encontram $48 \%$ de dominância de direita, $16 \%$ de dominância de esquerda e $36 \%$ balanceada. Analisando a dominância de perfusão, ou seja, observando qual das artérias coronárias é capaz de perfundir maior quantidade de solução salina, observam os seguintes resultados: $19 \%$ de dominância de dirieta, $69 \%$ de dominância de esquerda e $12 \%$ balanceada. O maior fluxo da artéria coronária esquerda em relação à direita indica que a quantidade de massa muscular suprida pelos dois vasos é importante na determinação da dominância.

Estudando a anatomia das artérias coronárias nos padrões normal e patológico, JAMES ${ }^{8}$ descreve minuciosamente o trajeto do ramo interventricular anterior, do ramo circunflexo e os ramos da artéria coronária direita. Observa que, em $90 \%$ dos casos, a artéria coronária direita cruza 0 sulco interventricular posterior para suprir aproximadamente metade da face diafragmática do ventrículo esquerdo. Nos $10 \%$ restantes, o ramo circunflxo da artéria coronária esquerda supre praticamente toda a face diafragmática desse ventrículo.

Descrevendo a anatomia das artérias coronárias em 125 corações humanos, PENTHER et alii 10 verificam que, em $10 \%$ dos casos, a artéria coronária esquerda é dominante,ou seja, ela dá origem ao ramo interventricular posterior e que, em $90 \%$ dos casos restantes o ramo interventricular posterior é originado da artéria coronária direita. Neste grupo estão incluídos dois tipos de circulação: dominância de direita e balanceada. Encontram, também, em $5 \%$ dos casos, um ramo interventricular posterior curto, que corre na porção proxial do sulco interventricular posterior.

Mais recentemente, em 1988, BAPTISTA et alii 2 descrevem o ápice do coração e seu suprimento sangüíneo em 81 corações normais ( 56 homens e 25 mulheres), através de estudo radiológico. O aspecto mais importante é a nomenclatura estabe- lecida para mais de 25 ramos das artérias coronárias, que pode servir de base para o estudo morfológico da irrigação cardíaca. Relatam que em $86,4 \%$ dos casos o ramo interventricular anterior forma uma alça ao redor do ápice, verificando diferenças entre homens e mulheres, sendo mais freqüente nestas.

Analisando vários trabalhos clássicos na literatura sobre as artérias coronárias e, somando-se a eles, conhecimentos anátomo-clínicos, acreditamos ser de grande importância estudar detalhadamente a anatomia das artérias coronárias.

O objetivo do nosso trabalho é determinar o tipo de circulação e predominância das artérias coronárias. Some-se o caráter pioneiro que tal pesquisa tem no Brasil, possibilitando dados estatísticos específicos para a população brasileira, uma vez que, como sabemos, devido à grande miscigenação, a população brasileira apresenta um caráter sui generis, para a qual não se deve aplicar estatísticas internacionais, exceto na ausência de outros dados. Visando complementar este trabalho, realizamos estudo morfométrico dos ramos que determinam a dominância, para estabelecer suas possiveis correlações anátomo-funcionais.

\section{MATERIAL E MÉTODOS}

Utilizamos 50 corações de adultos (17 a 80 anos) de ambos os sexos ( 35 masculino e 15 feminino), de diferentes raças ( 28 da raça branca, 21 da raça negra e 1 da raça amarela). obtidos de necropsia no Serviço de Verificação de Óbitos da capital. Após a obtenção dos corações, procedemos a:

1) Lavagem da peça em água corrente.

2) Cateterização das artérias coronárias a partir de seus óstios na aorta.

3) Injeção de solução fisiológica nas artérias coronárias para a eliminação de resíduos de sangue.

4) Injeção de $10 \mathrm{ml}$ de solução de gelatina corada pelo cinábrio sob pressão constante nas artérias cateterizadas.

5) Ligadura da artéria ao redor do seu óstio com fio de algodão $2-0$, visando evitar o refluxo da gelatina injetada.

6) Injeção de $10 \mathrm{ml}$ de gelatina corada pelo azul xadrez no óstio de abertura do seio coronário, visando facilitar a distinção entre artéria e veia durante a documentação fotográfica.

7) Lavagem da peça em água corrente por 10 minutos para endurecimento da gelatina.

8) Imersão da peça em solução de formalina a 
FALCIJÚNIOR, R.; CABRAL, R. H.; PRATES, N. E. V. B. - Tipos de circulação e predominância das artérias coronárias em coraçōes de brasileiros. Rev. Bras. Cir. Cardiovasc., 8(2):152-162, 1993.

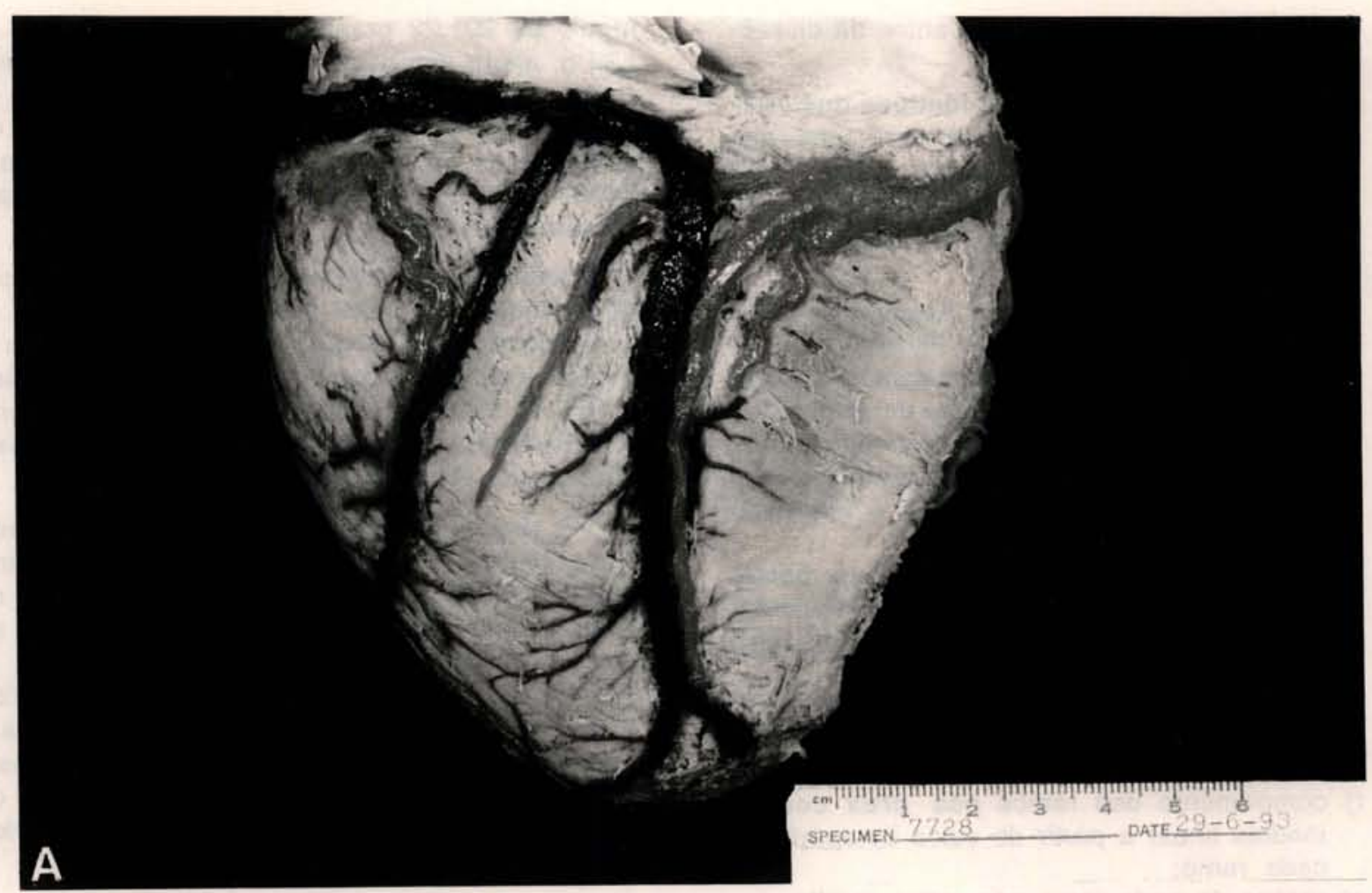

B

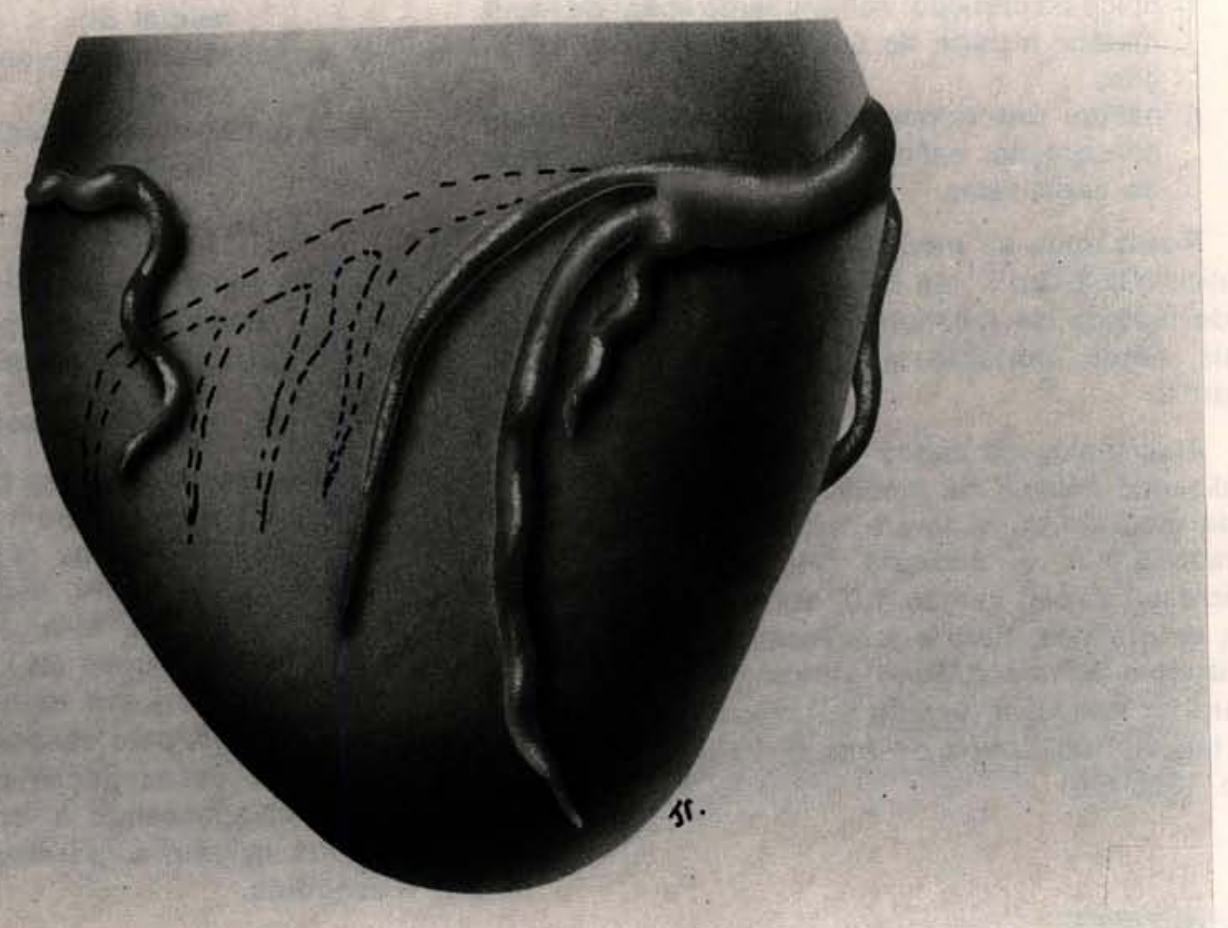

Fig. 1- A) Fotografia de coraçăo apresentando dominância de direita. B) Esquema representativo da fotografia, indicando os possíveis ramos pós crux cordis. 
FALCI JÚNIOR, R.; CABRAL, R. H.; PRATES, N. E. V. B. - Tipos de circulação e predominância das artérias coronárias em coraçōes de brasileiros. Rev. Bras. Cir. Cardiovasc., 8(2):152-162, 1993.

$10 \%$, por no mínimo sete dias antes da dissecção.

9) Dissecção macroscópica cuidadosa das artérias coronárias e seus ramos, bem como das veias cardíacas e suas tributárias, dando ênfase à sua distribuição na fase diafragmática, visando identificar o tipo de circulação.

10) Estudo morfométrico:

a) peso cardíaco: medido por balança de prato (técnica industrial Oswaldo Filizola) com divisão de um grama. (Padronizamos a pesagem com a retirada das peças da solução de formol uma hora antes do procedimento);

b) altura ventricular: distância linear entre o óstio da artéria coronária esquerda e ápice do coração;

c) comprimento do ramo interventricular posterior: medida linear do ramo interventricular posterior no seu trajeto no sulco interventricular posterior;

d) calibre do ramo interventricular posterior: calibre desse ramo no seu ponto médio;

e) número de ramos pós crux cordis;

f) comprimento dos ramos pós crux cordis: medida linear a partir do sulco coronário de cada ramo;

g) calibre de cada ramo pós crux cordis: calibre medido no ponto médio de cada ramo;

h) número de ramos que ultrapassam o ápice do coração;

i) comprimento dos ramos que ultrapassam o ápice do coração: comprimento linear do ramo medido a partir do ápice anatômico do coração;

j) calibre dos ramos que ultrapassam o ápice do coração: calibre medido no ponto médio de cada ramo.

Realizamos as medições com paquímetro eletrônico "Digit-Cal II" da Brown and Sharpe, na unidade métrica de milímetro, com duas casas decimais, tendo, portanto, a precisão de dez micrômetros.

Analisamos os dados obtidos estatisticamente, realizando cálculo da média e desvio padrão para cada medição do software especializado "Epi-Info" versão 5.0 A, o software "Harvard Graphics" e "Microsoft Excel" versão 4.0, obtendo, através destes, os gráficos. Para o processamento do texto foi utilizado o "Microsoft Word" versão 5.0 e o "Microsoft Word for Windows" versão 2.0 e, para a confecção de tabelas, utilizamos o software "Flow Chart" versão 1988-89.

\section{RESULTADOS}

Os 50 corações estudados apresentam um peso médio de 291,22 gramas (g) variando de $143 \mathrm{~g}$ a $533 \mathrm{~g}$; altura ventricular média de 97,20 milímetros (mm) variando de $77,08 \mathrm{~mm}$ a $130,18 \mathrm{~mm}$ e o comprimento do sulco interventricular posterior com variação de $58,12 \mathrm{~mm}$ e 104,35 mm (média de $76,79 \mathrm{~mm}$ ). Estas medidas nos dão uma idéia do tamanho das peças.

Dessas peças, $72 \%$ das observações são de dominância de direita (Figura $1, A$ e B), o que corresponde a 36 peças; 8 peças apresentam dominância de esquerda (Figura 2, A e B), equivalente a $16 \%$ do total e a circulação balanceada (Figura 3 , A e B) ficou com $12 \%$, correspondente a 6 peças anatômicas (Gráfico 1).

O ramo interventricular posterior apresentou comprimento médio de $48,74 \mathrm{~mm}$, sendo o comprimento máximo $82,62 \mathrm{~mm}$ e o mínimo $12,32 \mathrm{~mm}$. Para estudo dos ramos que ultrapassam a crux cordis adotamos a nomenclatura seguida por BAPTISTA et alii ${ }^{2}$ e FALCI JÚNIOR \& PRATES 6 , segundo a qual utilizamos a letra $\mathbf{M}$ seguida de números romanos consecutivos para os ramos da artéria coronária direita que ultrapassam a crux crodis e irrigam a parede posterior do ventrículo esquerdo:

$1^{\circ}$ ramo) $\mathrm{M}-\mathrm{I}$ : ramo ventricular posterior esquerdo medial I;

$2^{\circ}$ ramo) $\mathrm{M}$-II: ramo ventricular posterior esquerdo medial II;

$3^{\circ}$ ramo) $\mathbf{M}$-III: ramo ventricular posteriot esquerdo medial III:

$4^{\circ}$ ramo) M-IV: ramo ventricular posterior esquerdo medial IV;

5 ramo) $\mathbf{M}-\mathbf{V}$ : ramo ventricular posterior esquerdo medial $\mathrm{V}$.

O número médio de ramos encontrado foi 2,27.

De maneira análoga, utilizamos a letra $L$ seguida do número romano para designar a artéria do ramo circunflexo que ultrapassa a crux cordis:

L-I: ramo ventricular posterior direito lateral I.

Dentre as 36 peças com dominância de direita, $67 \%$ (24) apresentaram dois ramos (M- e $\mathbf{M}-\mathrm{II}$ ) ultrapassando a crux cordis, $42 \%$ (15) apresentaram três ramos (M-I, M-II e $\mathbf{M}$-III), $14 \%$ (5) quatro ramos (M-I, M-II, $\mathbf{M}-\mathbf{I I I}$ e $\mathbf{M}-\mathbf{I V}$ ) e apenas $6 \%$ (2) tinham cinco ramos (M-I, $\mathbf{M}-\mathbf{I I}, \mathbf{M}-\mathbf{I I I}, \mathbf{M}-\mathbf{I V}$ e $\mathbf{M}-\mathbf{V}$ ) ultrapassando essa estrutura (Figura 4). Deve-se recordar que, pelo conceito de dominância de direita, todas essas peças possuem pelo menos um ramo ultrapassando a crux cordis e todos esses ramos irrigam a parede posterior do ventrículo esquerdo.

Estudo análogo foi procedido para os casos de dominância de esquerda, no qual verificamos que 
FALCIJÚNIOR, R.; CABRAL, R. H.; PRATES, N. E. V.B. - Tipos de circulação e predominância das artérias coronárias em coraçōes de brasileiros. Rev. Bras. Cir. Cardiovasc., 8(2):152-162, 1993.
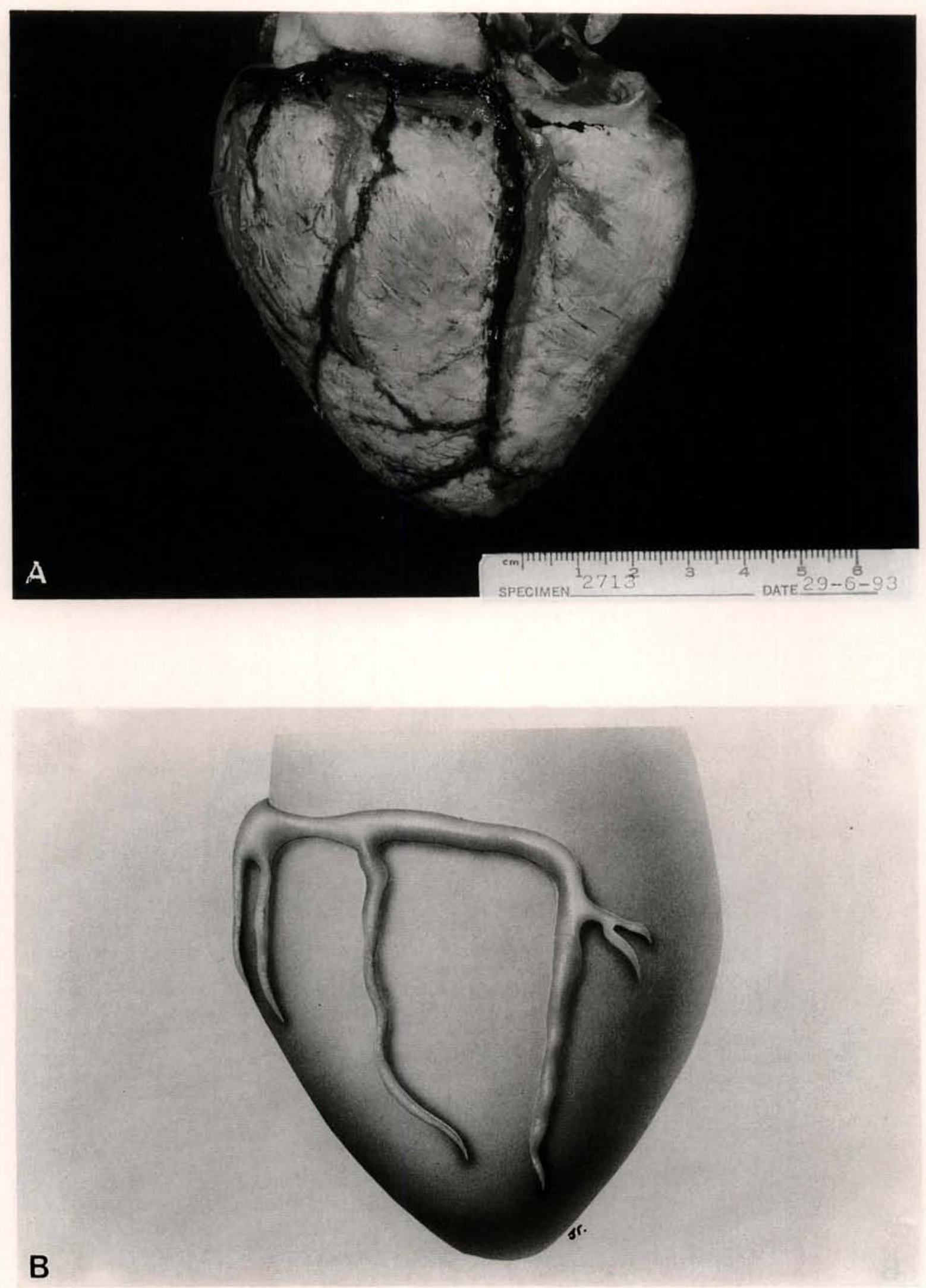

Fig. 2- A) Fotografia de coraçăo apresentando dominância de esquerda. B) Esquema representativo da dominância de esquerda. 


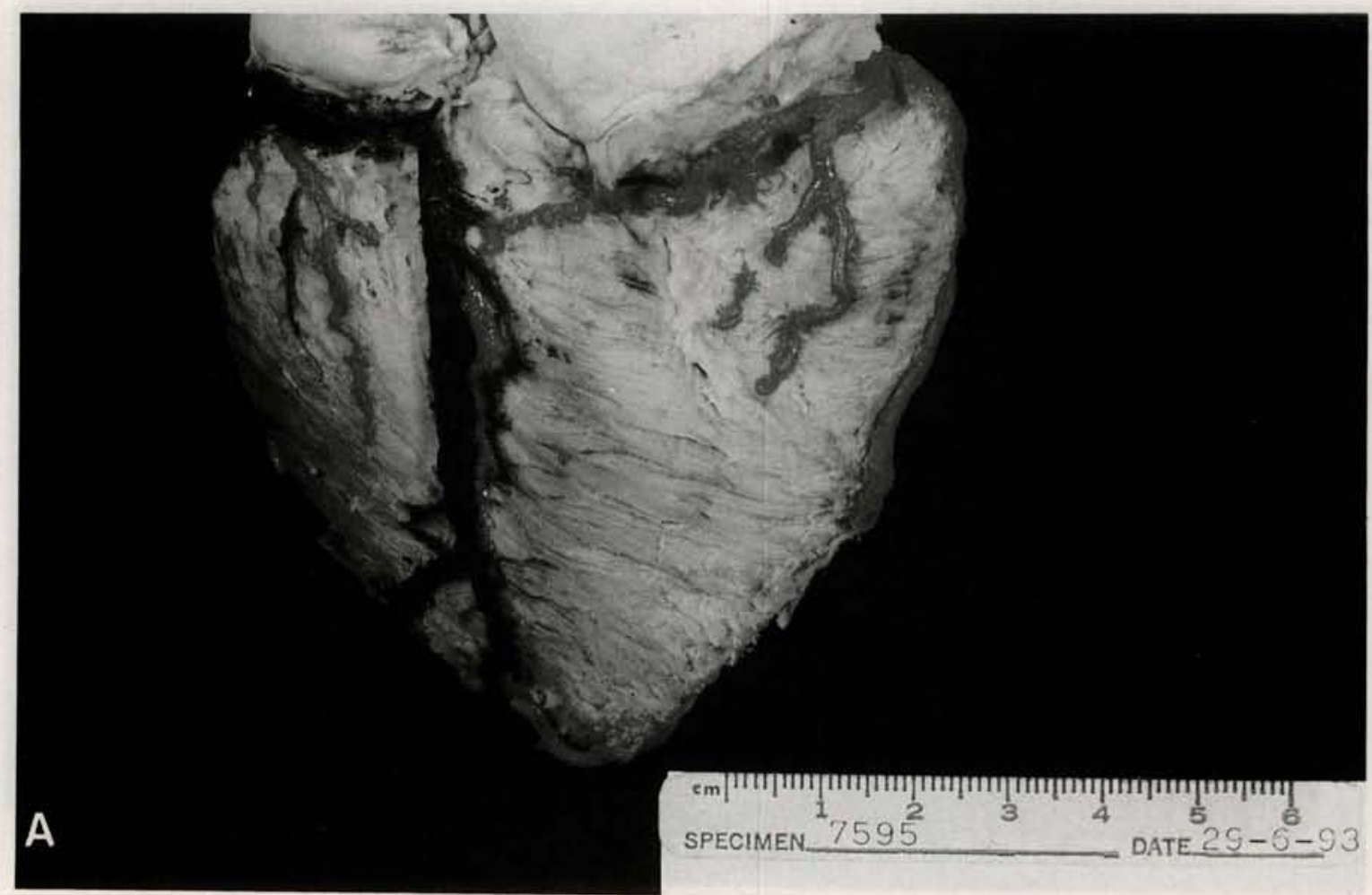

B

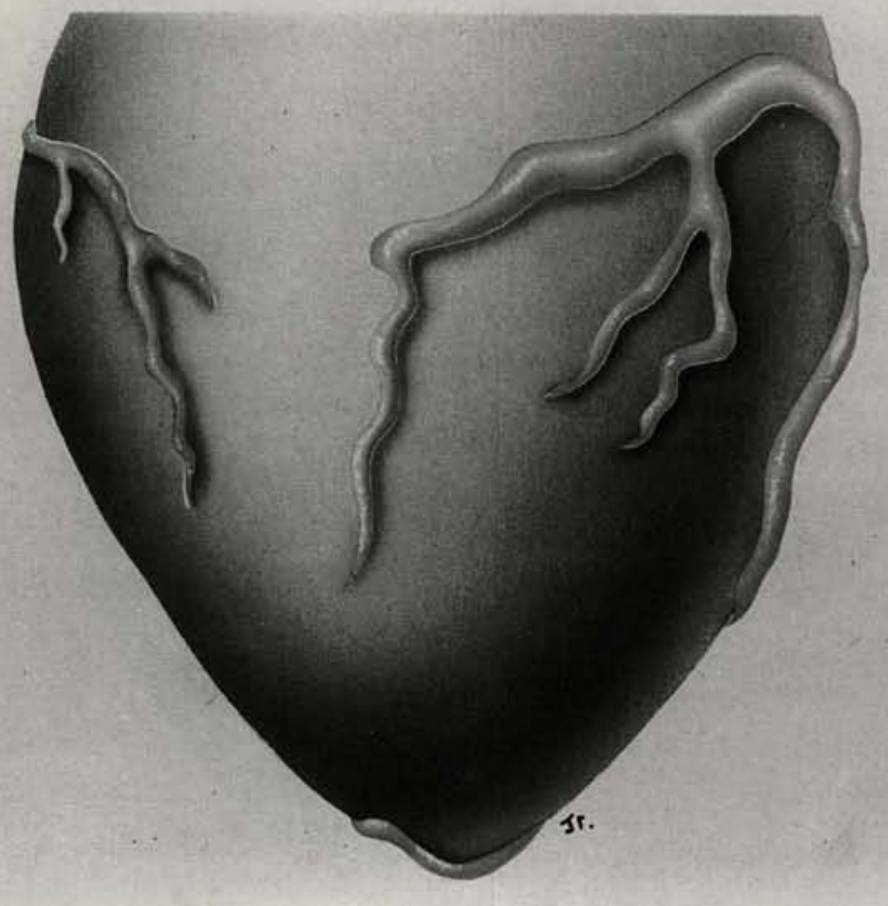

Fig. 3 - A) Fotografia de coração evidenciando circulaçăo tipo balanceada; B) Esquema representando o tipo de circulação balanceada. 
FALCI JÚNIOR, R.; CABRAL, R. H.; PRATES, N. E. V.B. - Tipos de circulaçăo e predominância das artérias coronárias em corações de brasileiros. Rev. Bras. Cir. Cardiovasc., 8(2):152-162, 1993.

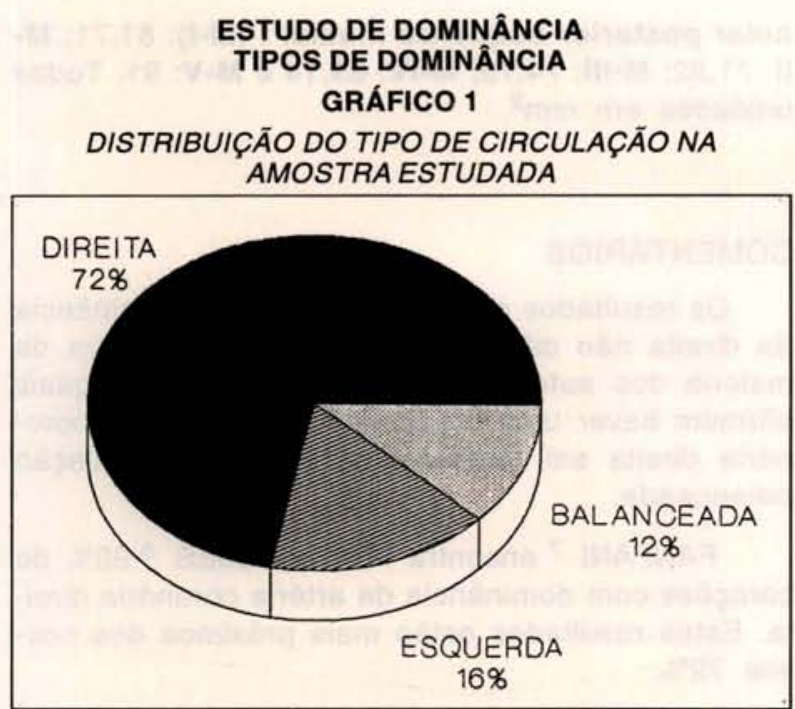

em $16 \%$ dos casos (2 peças de um total de $8 \mathrm{com}$ dominância de esquerda), o ramo circunflexo da artéria coronária esquerda dá, além do ramo interventricular posterior, um ramo terminal que ultrapassa a crux cordis (L-I). Nesses casos, temos uma grande massa de músculo cardíaco dependente de uma única artéria. No Gráfico 2, obtivemos esses valores em relação à totalidade das peças (50).
Realizamos, ainda, a correlação numérica entre o comprimento do ramo interventricular posterior e o sulco interventricular posterior, obtendo um número entre zero e um, que nos dá uma idéia do comprimento relativo desse vaso. Com este resultado, tentamos estabelecer relação estatística entre este valor e o fato de o ramo interventricular anterior passar para a região posterior do coração. Em $50 \%$ (25) das observações o ramo interventricular anterior ultrapassa o ápice do coração e atinge a região posterior (Figura 5), enquanto em apenas 4\% (2) dos casos o ramo interventricular posterior passa para a região anterior. Nas 25 peças cujo ramo interventricular anterior ultrapassa o ápice do coração, a média da relação entre esse ramo e o sulco interventricular posterior foi de 0,61 , enquanto que, nos restantes, a média encontrada foi de 0,68 , provavelmente demonstrando que esse talvez não seja o único fator que determine a passagem desse ramo para a região posterior.

Nos corações que apresentam dominância de direita, calculamos o produto comprimento $\mathbf{X}$ calibre para cada ramo proveniente da artéria coronária direita, ou seja, ramo interventricular posterior e ramos ventriculares posteriores esquerdos mediais I, II, III, IV e V, sempre que presentes. Com essa medida, tentamos estabelecer a importância relati-

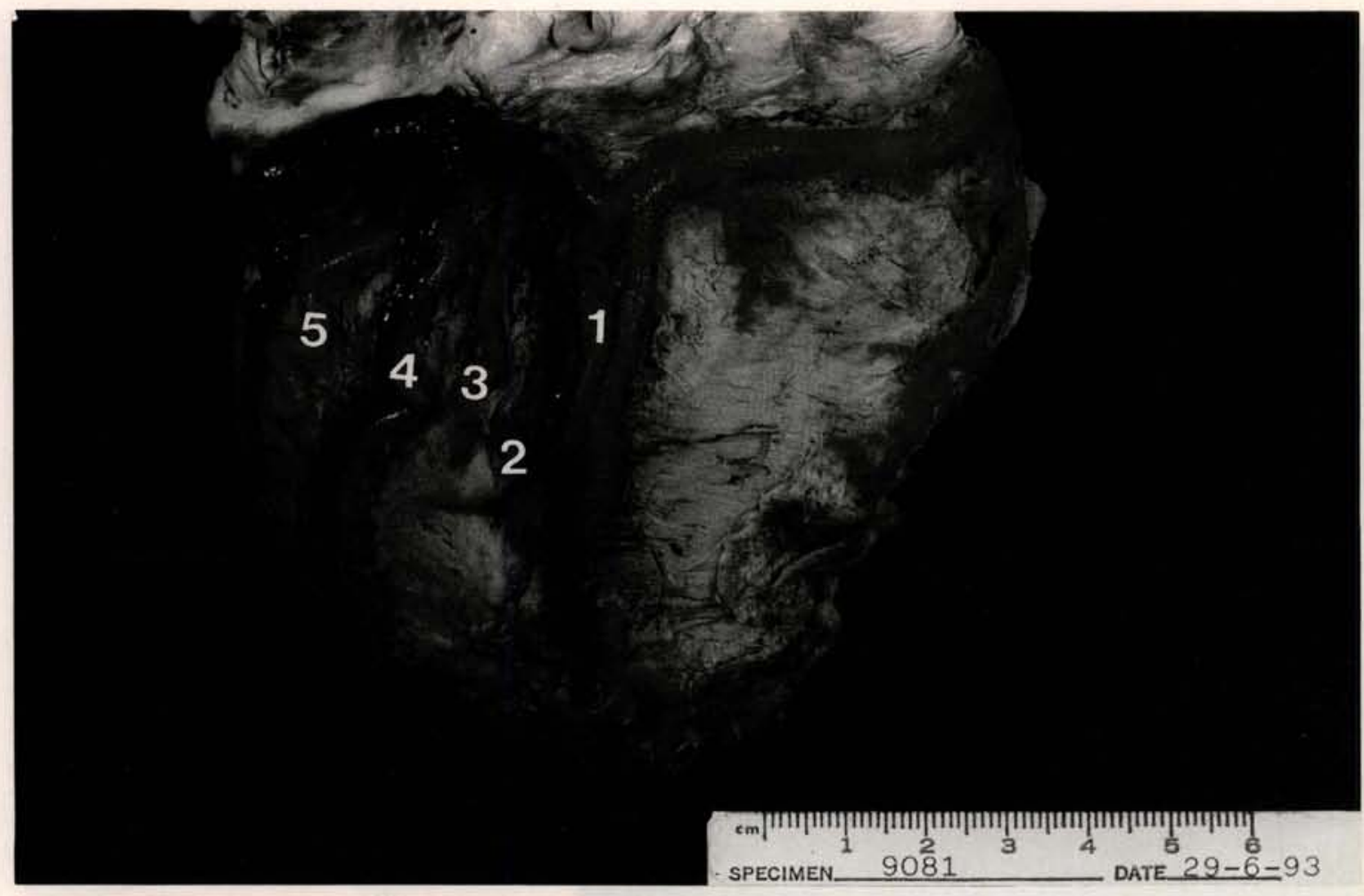

Fig. 4 - Fotografia de coração apresentando circulação de dominância de direita. Notar os cinco ramos que ultrapassam a crux cordis: M-I (1), MII (2), M-III (3), M-IV (4) e M-V (5). 
FALCI JÚNIOR, R.; CABRAL, R. H.; PRATES, N. E. V. B. - Tipos de circulação e predominância das artérias coronárias em coraçōes de brasileiros. Rev. Bras. Cir. Cardiovasc., 8(2):152-162, 1993.

\section{ESTUDO DE DOMINÂNCIA \\ RAMOS PÓS CRUX CORDIS \\ GRÁFICO 2}

PRESENCA DE RAMOS PÓS CRUX CORDIS EM RELAÇĀO AO TOTAL DE PEÇAS (50 CORAÇÖES).

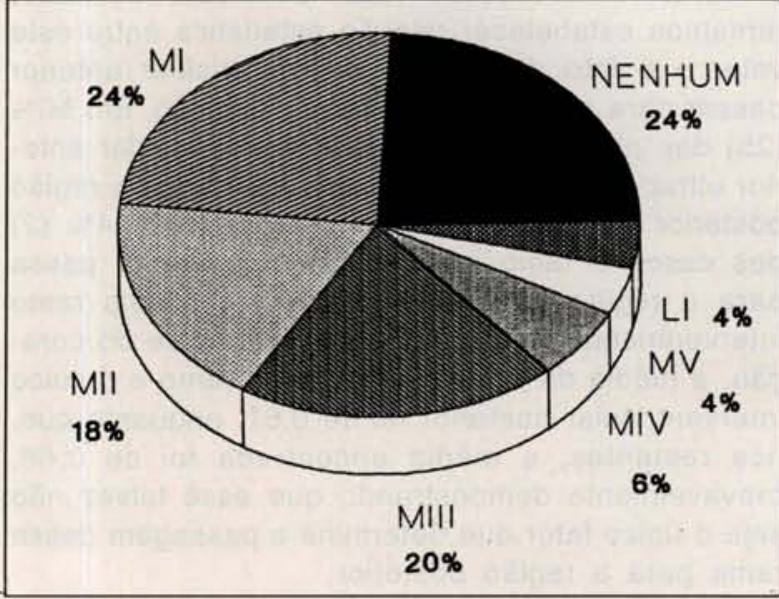

va de cada ramo no suprimento sangüíneo da região posterior do ventrículo esquerdo, no caso de dominância de direita.

A média obtida para cada ramo é de: ramo interventricular posterior: 160,88 ; ramo ventri- cular posterior esquerdo medial I (M-I): 81,71; MII: 71,82 ; M-III: 74,75 ; M-IV: 89,14 e M-V: 91 . Todas unidades em $\mathrm{mm}^{2}$.

\section{COMENTÁRIOS}

Os resultados obtidos em relação à dominância de direita não diferem de maneira significativa da maioria dos autores da literatura citada, os quais afirmam haver uma predominância da artéria coronária direita em relação à esquerda e circulação balanceada.

FANFANI ${ }^{7}$ encontra $77 \%$ e JAMES ${ }^{8} 90 \%$ de corações com dominância da artéria coronária direita. Estes resultados estão mais próximos dos nossos $72 \%$.

A dominância de esquerda e a circulação balanceada referidas em proporções próximas pelos diversos autores, estão de acordo com nossos achados para a população brasileira (Quadro 1).

Dos autores citados, apenas MAY ${ }^{9}$ refere-se ao número de ramos que ultrapassam a crux cordis em casos de dominância de direita, relatando uma média de 1,8 ramos, o que está próximo da média por nós encontrada de 2,2 ramos. Não há reltatos

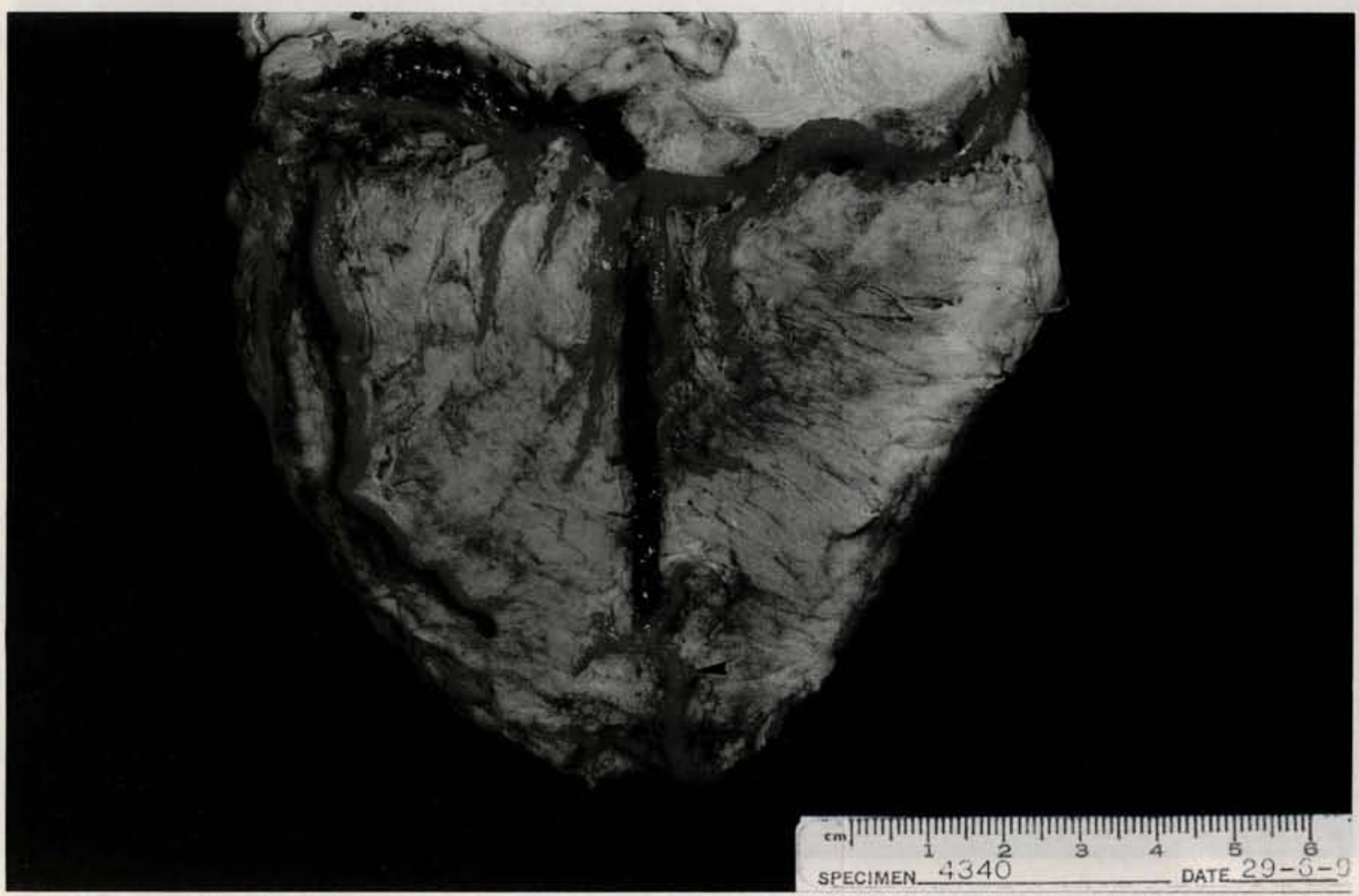

Fig. 5 - Fotografia de coração evidenciando o ramo interventricular anterior ultrapassando o ápice do coração e atingindo a região posterior (seta). 
FALCI JÚNIOR, R.; CABRAL, R. H.; PRATES, N. E. V.B. - Tipos de circulação e predominância das artérias coronárias em corações de brasileiros. Rev. Bras. Cir. Cardiovasc., 8(2):152-162, 1993.

QUADRO 1

RESULTADOS OBTIDOS POR DIFERENTES AUTORES QUANTO AO TIPO DE CIRCULAÇÃO CORONÁRIA.

\begin{tabular}{|c|c|c|c|c|}
\hline AUTOR & DIREITA & & BALANCEADA & ESQUERDA \\
\hline Campbell & $68 \%$ & & $18 \%$ & $14 \%$ \\
\hline May & $55 \%$ & & $9 \%$ & $36 \%$ \\
\hline Schlesinger & $48 \%$ & & $34 \%$ & $18 \%$ \\
\hline Blumgart et alii & $40 \%$ & & $40 \%$ & $20 \%$ \\
\hline Zoll & $50 \%$ & & $16 \%$ & $44 \%$ \\
\hline Fanfani & $77 \%$ & & $7 \%$ & $16 \%$ \\
\hline Vasko et alii & $48 \%$ & & $36 \%$ & $16 \%$ \\
\hline James & & $90 \%$ & & $10 \%$ \\
\hline Penther et alii & & $90 \%$ & & $10 \%$ \\
\hline Falci Jr. et alii & $72 \%$ & & $12 \%$ & $16 \%$ \\
\hline
\end{tabular}

na literatura sobre comprimento e calibre desses ramos.

BAPTISTA et alii ${ }^{2}$ relatam que em $86,4 \%$ dos casos o ramo interventricular anterior forma uma alça ao redor do ápice atingindo a face diafragmática do coração. Em nosso material, em $50 \%$ dos casos este fato foi comprovado, o que demonstra a importância desse ramo, cuja oclusão pode provocar infartos de grande extensão.

O nosso estudo fornece dados sobre a dominância anatômica das artérias coronárias; contudo, cumpre-nos complementar através de levantamentos obtidos pelas cinecoronariografias. De acordo com VASKO et alii ${ }^{14}$, a dominância de perfusão que reflete uma predominância funcional das artérias coronárias mostra uma inversão de valores com $69 \%$ de dominância de esquerda, o que significa que a dominância anatômica não corresponde exatamente à dominância funcional.

Cabe-nos ressaltar, ainda, que a dominância da direita é a manutenção do padrão fetal de circulação e que, mesmo nesses casos, existe um predomínio da artéria coronária esquerda devido a que esta artéria irriga paredes cardíacas com maior massa muscular.

\section{CONCLUSÕES}

1) Em corações de brasileiros, a dominância de direita é consideravelmente maior que a dominância de esquerda e circulação balanceada.

2) Na metade dos corações estudados encontramos um ramo interventricular anterior que ultrapassa o ápice cardíaco e atinge a região posterior.

3) Não existe correlação estatisticamente significativa entre o comprimento do ramo interventricular posterior e os ramos do ramo interventricular anterior que ultrapassam o ápice do coração.

4) Nos corações com dominância de direita, a maioria apresenta mais de um ramo ultrapassando a crux cordis, com um máximo de cinco ramos.

5) Nos corações com dominância de esquerda, a maioria não apresenta ramos ultrapassando a crux cordis.

6) Nos corações com dominância de direita, os maiores ramos são o interventricular posterior, o $\mathbf{M}-\mathbf{V}$ e o $\mathbf{M}-\mathbf{I V}$, quando presentes, em ordem decrescente de tamanho. Esse fato demonstra a importância da artéria coronária direita na irrigação de toda a parede posterior do ventrículo esquerdo. 
FALCIJÚNIOR, R.; CABRAL, R. H.; PRATES, N. E. V.B. - Tipos de circulação e predominância das artérias coronárias em corações de brasileiros. Rev. Bras. Cir. Cardiovasc., 8(2):152-162, 1993.

RBCCV $44205-210$

FALCI JÚNIOR, R.; CABRAL, R. H.; PRATES, N. E. V. B. - Predominance of the coronary arteries of the brazilian hearts: morphometric study. Rev. Bras. Cir. Cardiovasc., 8(2):152-162, 1993.

ABSTRACT: The type of circulation and the predominance of the coronary arteries have a great interest in Cardiology as well as in Cardiac Surgery. There is a close relationship between the type of circulation and the risk of myocardial injuries after coronary obstruction. We carried out 50 normal hearts ( 35 males and 15 females) of 28 caucasian and 22 non-caucasian individuals. The arteries were injected with colored gelatine. The average cardiac weight was $291 \mathrm{~g}$ and the ventricular length was $97 \mathrm{~mm}$. The right coronary predominance was the most common pattern of distribution $(72 \%)$ and is followed in incidence by a left coronary predominance $(16 \%)$ and least frequently by a balanced circulation $(12 \%)$. The incidence of branches crossing the crux cordis ranged from 1 to 5 (average 2,2) in right coronary predominance. On the other hand, in the left coronary predominance we found one branch in two hearts. The most frequent branch reaching the diaphragmatic from the sternocostal surface was the interventricularis anterioris ramus $(50 \%)$.

DESCRIPTORS: coronary arteries, morphology; coronary arteries, anatomy.

AGRADECIMENTO: Ao Sr. Sérgio Spezzia, Sr. Hélio Francisco de Souza e Sr. Argemiro Falcett Júnior, pela documentação científica. Ao Sr. Divino Geraldo da Silva, José Adão Mendes e Edson Dantos da Silva, pela ajuda na coleta do material. Ao Prof. Dr. Clóvis Takiguti e Sr. Ivaldo, pela interpretação dos dados estatísticos. Ao Prof. Dr. Fábio B. Jatene, pela colaboração no uso dos recursos técnicos do Museu anatômico-cirúrgico. Ao Departamento de Patologia da Faculdade de Medicina da Universidade de São Paulo, pelo empréstimo dos computadores.

\section{REFERÊNCIAS BIBLIOGRÁFICAS}

1 BANCHI, A. - Morfologia delle arteriae coronariae cordis. Arch. Ital. Anat, Embriol., 3: 87-164, 1904.

2 BAPTISTA, C. A. C.; DIDIO, L. J. A.; DAVIS, J. T.; TEOFILOVSKY-PARAPID, G. - The cardiac apex and its superficial blood supply. Surgical Radiol. Anat. 10: 151-160, 1988.

3 BLUMGART, H. L.; SCHLESINGER, M. J.; DAVIS, D. - Studies on the relation of the clinical manifestation of angina pectoris, coronary thrombosis, and myocardial infarctation to the pathologic findings, with particular reference to the significance of the collateral circulation. Am. Heart J. 19: 1-91, 1940.

4 CAMPBELL, J. S. - Stereoscopic radiography of the coronary system. Quart. J. Med., 22: 247-268, 1929.

5 EHRICH, W.; de la CHAPELLE, C.; COHN, A. E. Anatomical ontogeny: a study of the coronary arteries. Am. J. Anat., 49: 241-282, 1931.

6 FALCI JÚNIOR, R. \& PRATES, N. E. V. B. - Anatomia das artérias coronárias. Rev. Med. (São Paulo) 72, 1993 (No prelo).
7 FANFANI, M. - Richerche sulle varianti del circolo coronarico del cuore, con particolare riguardo alla irrorazione del setto interventricolare e ai problemi del circolo collaterale. Arch. Vecchi Anat. Patol., 19: 779-795, 1953.

8 JAMES, T. N. - Anatomy of the coronary arteries in health and disease. Circulation, 32: 1020-6033, 1965.

9 MAY, A. M. - Surgical anatomy of the coronary arteries. Dis. Chest., 38: 645-657, 1960.

10 PENTHER, P.; BARRA, J. A.; BLANC, J. J. - Étude anatomique descriptive des gros troncs coronariens et des principales collatérales épicardiques. Nouv. Presse Med., 5: 71-75, 1976.

11 SCHLESINGER, M. J. - Relation of the anatomic pattern to pathologic conditions of the coronary arteries. Arch. Pathol., 30: 403-415, 1940.

12 SCHLESINGER, M. J. - Significant variations in the anatomic pattern of the coronary vessels. A. A. A. S., 13: $61,1940$.

13 SPADA, D.; PICCALUGA, A.; SCHLICH, G. - Studi di anatomia radiologica e di fisiopatologica del sistema arterioso coronarico. Arch. Ital. Istol. Pastol., 32: 381-397, 1958.

14 VASKO, J. S.; GUTELIUS, J.; SABISTON Jr., D. C. A study of predominance of human coronary arteries determinated by arteriographic and perfusion technics. Amer. J. Cardiol., 8: 379-384, 1961.
15

16
WHITTEN, M. B. - The relation of the distribution and structure of the coronary arteries to myocardial infarctation. Arch. Intern. Med., 45: 386-400, 1930.

ZOLL, P. M. - Normal and pathological anatomy of the coronaries. Trans. Amer. Coll. Cardiol., 1: 29-43, 1951. 